\title{
Using Organizational Policy to Create a Positive and Supportive Environment: An Example of Women and HIV Prevention
}

\author{
Nicole Britten, Andrew Papadopoulos* \\ Department of Population Medicine, University of Guelph, Guelph, Canada \\ *Corresponding author: apapadop@uoguelph.ca
}

Received January 29, 2013; Revised March 16, 2013; Accepted April 01, 2013

\begin{abstract}
Making meaningful contact with marginalized populations is a challenge that public health practitioners continually face. Vulnerable populations that are targeted in health promotion initiatives are frequent users of community-based organizations, making these agencies an important point of contact. The relationships communitybased organizations have with vulnerable populations are under-utilized for developing and disseminating health messages. Using the example of women and HIV prevention, this paper argues that amending policy within community organizations can be an effective method to improve access to health information and resources, and elicit positive change. Furthermore, greater collaboration and knowledge exchange between public health agencies and community service providers will ensure organizations have the capacity to implement evidence-based policies that foster an environment where long-term positive change can occur.
\end{abstract}

Keywords: organizational policy, policy networks, cross-sector collaboration, women and HIV, marginalized populations, community organizations

\section{Introduction}

It is well understood that modern day health problems are complex, and innovative solutions requiring the involvement of numerous stakeholders are necessary. Further, it is now widely accepted that the social determinants of health, an individual's social, economic, and physical environment play an integral role in shaping health and well being [1]. To this end, individuals who deal with factors such as poverty, violence, and homelessness are often the most vulnerable to health issues. However, they are also significantly marginalized, making it difficult to reach out to these populations. A woman's experience with HIV risk is no exception, as the social determinants significantly impact access to prevention information and resources [2,3,4]. Marginalized populations such as women at risk of HIV, are frequent users of community-based organizations in their communities, which can be defined as public or private non-profit organizations that work at a local level to provide programs and services with the goal of achieving equality across society. These organizations play an important role when targeting hard to reach populations.

This paper puts forward the argument that organizational policy is an effective tool to improve access to services amongst vulnerable populations such as women at risk of HIV, and bring about positive change. This argument is supported with a secondary argument; greater training within and greater collaboration and knowledge exchange among public health agencies and community-based agencies is necessary to ensure that organizations have the capacity to implement evidenceinformed policies that foster an environment where positive change can occur.

\section{Background}

\subsection{Women and HIV in Ontario}

Despite marked success in HIV prevention over the last 25 years, the number of individuals living with HIV/AIDS in Ontario continues to rise, with an increase of $67 \%$ in total diagnoses between 1999 and 2008 [5]. This increase can be attributed to both decreased mortality due to the increased success of medications, but also new infections among at-risk populations. HIV has often been stereotyped as a disease only experienced by men who have sex with men, or injection drug users. While these populations continue to be affected, Aboriginal peoples, people from countries where HIV is endemic, and women are also considered at risk [4].

It is particularly concerning that HIV positive diagnoses among women are increasing in both number and proportion. In 1985, less than two percent of new cases were women; today new diagnoses amongst women account for approximately $25 \%$ of new infections [6]. Women who are involved in sex work, women in prisons, female youth, women who use injection drugs, Aboriginal women, women from countries where HIV is endemic, and trans-women are considered to be more vulnerable to 
HIV infection [4]. According to the most recent report on HIV/AIDS in Ontario released by the Ministry of Health and Long Term Care over 50\% of new diagnoses amongst women can be attributed to heterosexual contact [6]. Currently, $15.6 \%$ of individuals living with HIV in Ontario are women, and approximately 300 women in Ontario test positive for HIV annually [6,7].

\subsection{Impact of the Social Determinants of Health}

The factors that are commonly known as the social determinants of health can be broadly characterized to include the social and economic environment, the physical environment and a person's individual characteristics and behaviours. More specifically in Canada, these factors include income and income distribution, education, unemployment and job security, employment and working conditions, early childhood development, food insecurity, housing, social exclusion, social safety network, health services, aboriginal status, gender, race, and disability [8]. These determinants are highly interconnected, and one's experience with each of the determinants will influence their experience with one or more of the others $[1,8]$.

Women are particularly affected by the way in which gender interacts with the other social determinants of health $[2,3,4]$. Gender is what shapes social norms regarding masculinity and femininity, and defines the social, economic and power imbalances that exist between men and women [4]. When a woman's gender intersects with sex, race, sexual orientation, age or class, the impact on a woman's health and well-being can be even greater. These factors can heavily influence a woman's experience with community-based organizations, and can have negative implications for accessing information, treatment and care relevant to their HIV prevention needs. Considering HIV prevention from a social determinants of health perspective can highlight a woman's individual experience with HIV, as well as inform organizational policy development that embodies a more holistic approach to HIV prevention.

\subsection{Stigma and Marginalization}

Stigma which can be defined as "processes of devaluing, labeling, and stereotyping that are manifested in the loss of status, unfair and unjust treatment, and social isolation of individuals or groups" [9,10], is perhaps the most significant barrier to women accessing testing, treatment or HIV prevention services [11]. HIV-related stigma has many negative consequences for women including social isolation, increased stress, depression and increased experiences of discrimination [12]. It plays a significant role in HIV risk as it intersects with other inequalities such as racism, sexism and homophobia [9]. Stigma among HIV-positive women is amplified as they are often assumed to be promiscuous or engaging in high-risk behaviours and are therefore deserving of their HIV status [11]. Further, gender norms that perceive women as mothers or caregivers exacerbate stigma directed toward positive women, as they may be viewed as failing to fulfill these social norms [12]. Experiences with stigma have been shown to be most significant amongst women who are members of other marginalized groups such as women of colour or ethnic minority $[11,12]$. While past studies have demonstrated increased stigmatization of certain populations such as intravenous drug users, or men who have sex with men, more recent findings from an Ontarian study suggest that interventions challenging stigma amongst these populations have been effective in reducing the self-perceived stigmatization within these groups [12].

\subsection{Stakeholder Engagement and Capacity Building}

Stakeholder engagement and community empowerment are critical to the success of any capacity building intervention $[13,14,15,16,17]$. The various components of capacity are not clearly defined, but in general encompass the skills, scarce resources, relationships, and organizational culture required to achieve an intended purpose effectively [18]. It is important to note that the term organizational capacity does not refer solely to financial resources.

Capacity is perhaps most easily understood using an example of a community health worker linked to the local healthcare delivery agency contributing to better health outcomes for women dealing with HIV. If an agency has positive relationships with women in the community and other local stakeholders such as their local AIDS Service Organization (ASO), has the necessary educational and support resources needed to deliver consistent and appropriate HIV messaging, is able to effectively interact with women living with or at risk of HIV and has the support of their agency to engage in such work, the community health worker has the capacity to perform their role successfully. Capacity can be measured at various levels including the individual, organizational, and community level.

While it is understood that public health agencies and community-based organizations are influential when building capacity, the target population plays an integral role in the building capacity of women to prevent HIV acquisition or transmission. Capacity is said to be a product of what the population of interest believes in, values, and does and although other parties are essential to the development of capacity, sustainable interventions involve the meaningful involvement of the target population at hand [18]. It is agreed upon within the literature that attempts to engage individuals within marginalized communities have resulted in limited success. Analysis of five community projects in the Canadian Health Promotion Contribution program revealed relatively low numbers and restricted range of participants, difficulties in recruiting and maintaining participants, declining rates of participation over time and limited target group influence [19]. Projects that were most successful reported control of projects by target group members rather than service agencies, highlighting the importance of community empowerment [19].

Efforts to develop community-based initiatives involving people living with HIV/AIDS (PLWHA) have also proven to be quite difficult because of the stigma associated with HIV, health-related challenges, lack of capacity to engage in research, other issues taking priority, and mistrust of resources. However, factors that facilitated the meaningful engagements of PLWHA included a valued lived experience, training and mentoring 
opportunities, financial compensation, trust building and accommodating PLWHA's needs [19].

Public policy creation exists in a complex environment and therefore, the creation of a policy network - a vast network of cross-sectoral stakeholders including government, public and private organizations, interest groups, and the target population is crucial for the development of healthy public policy [14,20]. This concept has been further characterized by Rhodes to describe a highly integrated and interdependent network, with a continual need for the exchange of resources [21]. Within a policy community, there will be recognition among the members that given the complexity of the issues, no one organization can solve the public problems [20]. Shared capacity will allow multiple organizations interested in serving the population to operate in an effective manner. A positive byproduct of a highly integrated and interdependent policy network is increased collaboration and information exchange among the network actors [21].

Within the women and HIV policy community, each stakeholder can have different levels of interaction with women at risk of, living with or affected by HIV, but regular interaction with the target population is an asset, as it will enhance trusting relationships needed to effectively engage women themselves. A literature review conducted by Jones and Sargeant revealed that community-based initiatives can lead to increased rates of testing, and healthy behaviour changes among at-risk populations [15]. This being said, in order for community-based organizations to deliver effective prevention interventions, the development of initiatives must be an evidenceinformed process that requires significant organizational capacity.

\section{Discussion}

Capacity must be enhanced in order to more effectively meet the prevention needs of women living with, at risk of or affected by HIV. Effective capacity development begins with three fundamental questions: 1) To what end to we need to develop this capacity? What will be its purpose? 2) Whose capacities need to be developed? Which groups or individuals need to be empowered? 3) What kinds of capacities need to be developed to achieve the broader development objectives [22]? Communitybased initiatives have proven promising in terms of HIV prevention, but greater system coordination and improved collaboration within the women and HIV policy network is required to adequately respond to the HIV prevention needs of women. Changes can occur at both the public health agency level and the community-based organization level to enhance collaboration and utilize the women and HIV policy network more effectively. Policy alternatives as a result of organizational reviews of internal and external activities, that focus on education and training, improvements in knowledge translation endeavors, as well as effective means of enhancing agency and network capacity is required. New and amended policies must be multifaceted solutions grounded in the social determinants of health, and challenge stigmatization and marginalization of women living with, at risk of or affected by HIV.
The role of community-based organizations is fundamental to ensure an effective capacity building strategy as they have the ability to inform, advocate, plan programs and deliver services to their targeted population [23]. Further, one of the greatest challenges faced in health promotion initiatives is making contact with target populations who are difficult to reach, and developing trusting relationships to ensure that the message is heard, and viewed as legitimate [1]. Community-based organizations are the conduit to vulnerable populations who make frequent use of social services, as they have relationships with their clients that are regular and ongoing, making them ideal candidates for message delivery [15]. It is essential that service providers optimize these relationships ensuring that they are meaningful, and are based on trust and respect.

While community-based organizations understand their role in dealing with health issues, decision makers within the organization may find it challenging to evaluate current policies appropriately, or implement new evidence-informed policies that have positive implications for the individuals whom they serve [23,24]. Many organizations are understaffed or underfunded and they lack the supply of, or the capacity to generate information to create evidence-informed policies [23]. On the contrary, public health agencies are better equipped to develop, implement and evaluate evidence-informed policies and programs given their financial and human resources, although they do not have the same intimate relationships with target populations. As with all effective partnerships, each party can bring to the planning table a skill or resource that the other does not have. Public health organizations can ensure the necessary resources to create policy and build organizational capacity to support service users, and community-based organizations can reach out to marginalized populations to involve them in the policy making process and greatly improve uptake of public health messages that are integrated into their agencies.

Change in policy is an effective tool to enhance capacity at an organizational level [25,26], and can play an important role in creating a supportive environment for women to address sensitive issues of concern such as HIV risk. Organizational policies have an immediate impact on the accessibility of an agency's services. Decision makers must be able to evaluate their current policies and procedures and recognize their ability to leverage policy instruments to solve issues or exploit opportunities. Further, decision makers within organizations may not realize that existing policies may have unintended negative effects for some of the clients whom they serve.

The integration of HIV prevention messages specific to women into waiting rooms, client rooms, or community events of existing network agencies that serve at risk women will greatly improve access to important information and resources [7]. The benefits of such a policy are twofold. First, it prevents feelings of shame associated with accessing services such as an ASO, by making information easily accessible in locations where at-risk women frequent. Secondly, it can also greatly increase awareness and understanding of HIV risk amongst women in the community, allowing for more communication about prevention. Having policies that ensure confidentiality and inclusiveness is equally as important to improve access to HIV prevention resources 
for women. While resources such as condoms may be available in many agencies who serve women considered to be at risk of HIV, a woman may feel embarrassed or judged for accessing them if they are located in visible places such as waiting rooms [7]. Adapting policy so that condoms are made available in washrooms or within washroom stalls where they can be accessed in privacy, can greatly improve the likelihood that women utilize available resources [7].

Organizations must possess a strong policy capacity to ensure a successful and ongoing policy development and review environment. Capacity within all levels of the organization, including at the individual level, at the operational level, and at the board level must be present and the resulting policies must be multidimensional [26]. Further, organizational structures, processes, and cultures must be supportive of policy development and enhancement. Without these capacities, organizations may attempt to develop or amend existing policy but may do so unsuccessfully.

It is recommended that organizations conduct an environmental scan to ensure internal and external activities are supportive of proactive policy development. This scan should include questions regarding the organizations goals and values, their relationship with other stakeholders in the policy network, their ability to support continuing professional development, their understanding of the impact current policies and procedures have on the women that they serve, and their skills and resources relevant to researching, developing, implementing and monitoring policies. Appropriate organizational culture will support external relations among organizations and create an environment where more effective relationships can be fostered.

\subsection{Education and Training}

Organizational policy should be integrated within longterm strategic plans and within shorter-term operational plans of agencies. These plans should allow for a significant education and training component. Continuing and ongoing education supported by the agency, would be required to ensure employees are adequately trained and have the necessary resources to acquire further information that can support women living with or at-risk of HIV. Employees of public health agencies and community-based organizations should be educated about a woman's unique experience with HIV-risk and have an understanding of the role the social determinants of health play in their experiences. Understanding the intersectional nature of these factors will facilitate the essential increase in awareness, education, and positive communication regarding the complexity of HIV risk. Education and training goes beyond the identification of such knowledge and skill enhancing programs, but incorporates the opportunity of individuals to participate in these training sessions, including financial and travel support. Individuals should be afforded adequate time to upgrade their skills without concern for their workplace workload. Free educational workshops offered by ASOs across Ontario regarding women and HIV, harm reduction, and working with marginalized populations should be better utilized. Interventions should be aimed at challenging stigma and marginalization of women living with, at risk of or affected by HIV to parallel successes of similar interventions with other at-risk populations.

\subsection{Knowledge Exchange}

More effective use of shared resources within the network will enhance network capacity to respond to the unique HIV prevention and support needs of women through knowledge exchange and evidence-informed policy development. Both public health agencies and community-based organizations play a critical role in this process because of their differing strengths and resources. Public health professionals are able to access and synthesize scientific evidence and provide tangible resources, while service providers from community-based organizations can share their experiences of working with women living with, at risk of, or affected by HIV to provide context to published literature and act as a voice for the target population. Stakeholder engagement is viewed as a critical component of successful public health interventions [13,14,15,16,17], yet the act of engaging marginalized populations is a continuous challenge for public health professionals [19]. Service providers who work with at risk women on a day-to-day basis should be increasingly involved in the policy process to help to include or as a minimum, act as proxy for the target population to ensure feasibility and acceptance of proposed solutions. An ongoing two-way dialogue between community-based organizations and women in the target population is critical to successful knowledge exchange, as it will have the positive effect of creating uptake from the target audience, as they are better able to identify emerging trends, and contribute to policy development, implementation and evaluation.

\subsection{Working within the Policy Network}

The interdependence of organizations within the policy network is fueled by continual interaction between network members and an ongoing need to exchange resources [21]. Within the women and HIV policy network, local public health agencies and organizations that have a strong research focus, provide a valuable knowledge base for policy making, but implementation of these policies is not possible without the inclusion of community-based organizations that will deliver programs and services to at-risk populations. Identifying organizational strengths within the policy network will facilitate increased collaboration of all of those involved. Ideally, the network should develop a working committee, with input from all members where roles and responsibilities can be identified. This will help ensure the efficient allocation of resources by decreasing overlap and maximizing the opportunity for resource exchange. Effective and consistent communication is essential in ensuring that the women and HIV policy network is able to sustain these interactions.

This approach will not be without its challenges as community-based organizations are typically under resourced and do not have the precedent of sharing scarce resources among partner agencies. Many of the community-based agencies that engage with this target market may have similar capacity given their origins and focus, and many gaps within the policy network may continue to exist, even through extensive collaboration. 
Engaging a vulnerable, hard-to-reach target market for a sustained period of time that would allow for meaningfully participation in the development of educational programs and services has proven very difficult to achieve [19]. Additional research regarding the challenges associated with sustained engagement of marginalized populations as well as the challenges of community-based research is necessary.

\section{Conclusion}

Community-based organizations are an under-utilized resource for ensuring evidence-informed HIV prevention policies and delivering prevention messages. For these organizations to be truly effective, they require an ongoing review of their organizational policies to ensure that these policies create a supportive and enabling environment.

Community-based organizations are the conduit to vulnerable populations, and increasing their involvement in the development and implementation of prevention initiatives provides the necessary community-level participation for sustainable positive change to occur $[14,24,25]$. These organizations can give women a voice in the policy making process, ensuring services are more inclusive and integrating acceptance that HIV is an issue that affects women into the community. This is a huge step forward in challenging stigma and discrimination, the greatest barriers to accessing services, treatment or support. In the bigger picture, using organizational policy to generate community support and educational messages will make these items more accessible.

Building organizational capacity to strengthen overall community capacity is necessary to respond to all multifaceted health issues of increasing complexity. Improving policy at an organizational and network level will facilitate the essential increase in awareness, education, and positive communication regarding health issues that precedes the adoption of healthier behaviours and new social norms. Improving partnerships between public health organizations and community-based organizations is crucial to ensure policies are improved accordingly. Moreover, creating effective partnerships based on complementary resources and need will allow public health practitioners to overcome the challenge of developing trusting relationships with target populations, and greatly increase uptake of health messages. Greater inclusion of the target audience in the development of these programs and educational materials will greatly enhance their success, ensure uptake, and create further awareness among the target community of these initiatives. Improving organizational policy is the necessary first step to foster an environment where meaningful, positive change can occur, and the health of our population can improve as a whole.

\section{Acknowledgement}

The authors would like to acknowledge the AIDS Committee of Guelph and Wellington County for their support, knowledge and guidance.

\section{Statement of Competing Interests}

The authors have no competing interests.

\section{References}

[1] Edberg, M., Essentials of Health Behaviour: Social and Behavioural Theory in Public Health, Jones and Bartlett Publishers, Sudbury, 2007.

[2] Leonard, L. Women and HIV prevention: A scoping review, HIV Prevention Research Team, 2007. [E-document] Available: http://www.medicine.uottawa.ca/epid/assets/documents/women_hi vprevention.pdf.

[3] McWilliam, S., Ontario Women and HIV/AIDS Working Group Literature Review: HIV Prevention and Women, Ministry of Health and Long Term Care, 2006. [E-book] Available: http://www.health.gov.on.ca/english/providers/pub/aids/reports/on tario_women_hivaids_working_group_literature_review_hi.pdf.

[4] Public Health Agency of Canada, Population-specific HIV/AIDS status report: Women, Public Health Agency of Canada, 2012. [Edocument] Available:

http://library.catie.ca/pdf/ATI-20000s/26407.pdf

[5] Ministry of Health and Long Term Care, Ontario HIV/AIDS Infection Rates, Ministry of Health and Long Term Care, 2010. [E-document] Available:

http://www.health.gov.on.ca/english/public/program/hivaids/gener al/charact_epidemic.html

[6] Remis, R.S., Swantee, C. and Liu, J, Report on HIV/AIDS in Ontario: 2009, Ministry of Health and Long Term Care, 2011. [Edocucment] Available:

http://www.ohemu.utoronto.ca/doc/PHERO2009_report_final.pdf

[7] AIDS Committee of Guelph and Wellington County, Women and HIV/AIDS Prevention Inititiative, AIDS Committee of Guelph and Wellington County, 2012. [E-document] Available: http://www.aidsguelph.org/women-and-hiv-prevention.

[8] Mikkonen, J. \& Raphael, D., Social Determinants of Health: The Canadian Facts, York University School of Health Policy and Management, 2010. [E-document] Available:

http://www.thecanadianfacts.org/The_Canadian_Facts.pdf

[9] Logie, C.H., James, I.J., Tharao, W. \& Loufty, M.R., "HIV, gender, race, sexual orientation, and sex work: a qualitative study of intersectional stigma experienced by HIV-positive women in Ontario, Canada," PLoS Med, 8(11), e1001124. November 2011.

[10] UNAIDS, Stigma and Discrimination Fact Sheet, 2003. [Edocument] Available: http://data.unaids.org/publications/FactSheets03/fs_stigma_discrimination_en.pdf

[11] Sandelowski, M., Lambe, C., \& Barroso, J., "Stigma in HIVpositive women," Journal of nursing scholarship: an official publication of Sigma Theta Tau International Honor Society of Nursing / Sigma Theta Tau, 36(2), 122-128. 2004.

[12] Loutfy, M. R., Logie, C. H., Zhang, Y., Blitz, S. L., Margolese, S. L., Tharao, W. E., et al. "Gender and ethnicity differences in HIVrelated stigma experienced by people living with HIV in Ontario, Canada," PloS one, 7(12), e48168. December 2012.

[13] Beeker, C., Guenther-Grey, C. \& Raj, A., "Community Empowerment Paradigm Drift and the Primary Prevention of HIV/AIDS," Social Science \& Medicine, 46(7): 831-842. 1998

[14] Smith, B. Public Policy and Public Participation Engaging Citizens and Community in the Development of Public Policy, Population and Public Health Branch Atlantic Region, Health Canada, 2003.

[15] Jones, J.E. \& Sargeant, S., Literature Review: The Impact of Community-Based HIV Prevention Interventions in Canada, Commonwealth Nations and the US, Public Health Agency of Canada, BC/Yukon Regional Office, 2009.

[16] Liberato, S.C., Brimblecombe, J., Ritchie, J., Ferguson, M. \& Conveney, J., "Measuring capacity building in communities: a review of the literature," BMC Public Health, 11:850-859. 2011.

[17] Zakus, J.D.L., "Resource Dependency and Community Participation in Primary Health Care," Social Science \& Medicine, 46(4-5), 475-494. 1998.

[18] Brinkerhoff, D.W., Capacity Development in Fragile States, ECDPM Masstricht, 2007, Discussion Paper 58D. 
[19] Boyce, W.F., "Disadvantaged persons' participation in health promotion projects: some structural dimensions," Social Science \& Medicine, 52, 1551-1564. 2001.

[20] Pal, L. (2010) Beyond Policy Analysis: Public Issue Management in Turbulent Times, Nelson Education, Toronto, 2006.

[21] Rhodes, R.A.W., "Understanding Governance: Ten Years On," Organization Studies, 28 (8). 1243- 1264. August 2007.

[22] United Nations Development Programme, Capacity Development: A UNDP Primer, United Nations Development Programme, 2009.

[23] Wilson, M.G., Rourke, S.B., Lavis, J.N., Bacon, J. and Travers, R., "Community capacity to acquire, assess, adapt, and apply research evidence: a survey of Ontario's HIV/AIDS sector," Implementation Science (6). 54-60. May.2011.

[24] Wilson, M.G, Lavis, J.N., Travers, R. and Rourke, S.B., "Community-based knowledge transfer and exchange: Helping community-based organizations link research to action," Implementation Science, 5(33), April 2010.

[25] Lavis, J.N., Oxman, A.D., Lewin, S and Fretheim, A., "Support Tools for evidence-informed health Policymaking (STP)," Health Research Policy and Systems, 7(Suppl 1), Dec 2009.

[26] Gleeson, D., Legge, D. O’Neill, D. and Pfeffer, M., "Negotiating Tensions in Developing Organizational Policy Capacity: Comparative lessons to be drawn," Journal of Comparative Policy Analysis, 13 (3). 237-263. 2011. 